\title{
EXISTENCE OF NON-TRIVIAL DEFORMATIONS OF SOME INSEPARABLE EXTENSION FIELDS
}

\author{
HIROSHI KIMURA
}

Dedicated to the memory of Professor Tadasi Nakayama

A deformation theory for rings and algebras was introduced recently by $\mathrm{M}$. Gerstenhaber [1]. Let $K$ be an extension of a field $k$, and $p$ denotes the characteristic. One of his results is that, if $K$ is separable over $k$, then it is rigid. It was conjectured in [1] that, if $K$ is not separable over $k$, then it is not rigid, and if it is further finitely generated, then an integrable element of $H_{c}^{2}(K, K)$ (see [2]) will be found in the image of $S q_{p}$. In this note we shall study the above conjecture in certain special case.

The author wishes to express his hearty thanks to Professor $K$. Masuda for his many helpful suggestion during the preparation of this paper.

1. Preliminaries. Let $K$ be an extension field of a field $k$ of characteristic $p$, and $V$ be the underlying vector space of $K$. Let $R$ and $S$ denote the power series ring $k[[t]]$ over $k$ in one variable $t$ and its quotient field $k((t))$ respectively, and let $V_{S}$ be $V \otimes_{k} S$.

Let a bilinear mapping $f_{t}: V_{S} \times V_{S} \longrightarrow V_{S}$ expressible in the form

$$
f_{t}(a, b)=a b+t F_{1}(a, b)+t^{2} F_{2}(a, b)+\cdots,
$$

where $F_{i}$ is a bilinear mapping defined over $k$, be a one-parameter family of deformations of $K$ considered as a commutative $k$-algebra. Then $f_{t}$ satisfies the conditions;

$$
\begin{aligned}
& f_{t}(a, b)=f_{t}(b, a) \\
& f_{t}\left(f_{t}(a, b), c\right)=f_{t}\left(a, f_{t}(b, c)\right)
\end{aligned}
$$

for all $a, b, c$ in $V_{S}$ and $F_{1}$ is an element of the group $Z_{c}^{2}(K, K)$ of commutative two-cocycles of $K$ with coefficients in $K$ (see [1]). Following [1], we say that $f_{t}$ is trivial if there is a nonsingular linear mapping $\Phi_{t}$ of $V_{S}$ onto itself of the form

Received March 20, 1967. 


$$
\Phi_{t}(a)=a+t \varphi_{1}(a)+t^{2} \varphi_{2}(a)+\cdots,
$$

where $\varphi_{i}$ is a linear mapping defined over $k$, such that

$$
f_{t}(a, b)=\Phi_{t}^{-1}\left(\Phi_{t} a \cdot \Phi_{t} b\right) .
$$

$K$ is rigid if and only if there is no non-trivial one-parameter family of deformations of $K$.

From now on, throughout this note, we assume $p \neq 0$.

The squaring operation is defined for every derivation $\varphi$ of $K$ over $k$ by $S q_{p} \varphi=-\frac{1}{p} \delta \varphi^{p}=\sum_{i=1}^{p-1} \frac{1}{p}{ }_{p} C_{i} \varphi^{p-i} \cup \varphi^{i}$, where $\delta$ denotes the coboundary operator and $U$ denotes the cup product. For example, if $p=2,3$, then

$$
S q_{2} \varphi=\varphi \cup \varphi,
$$

and

$$
S q_{3} \varphi=\varphi^{2} \cup \varphi+\varphi \cup \varphi^{2} .
$$

Gerstenharber proved in [1] that $S q_{p} \varphi \in Z_{c}^{2}(K, K)$. It is known ([1]) that, for any derivation $\varphi$ of $K$, there exists a one-parameter family $f_{t}$ of deformations of $K$ such that

$$
f_{t}(a, b)=a b+t S q_{p} \varphi(a, b)+t^{2} F_{2}(a, b)+\cdots \cdot
$$

2. Let $K$ be an inseparable extension field over $k$ such that there exists an inseparable algebraic element $\theta$ of exponent one over $k$ such that $\theta$ is not contained in $k\left(K^{p}\right)$. Let $f(X)=X^{m p}-a_{m-1} X^{(m-1) p}-\cdots-a_{1} X^{p}-a_{0}$ be the minimum polynomial of $\theta$ over $k$. Then there eixsts $a_{i} \neq 0,1 \leqq i \leqq m$, such that $i$ is not divisible by $p$ (where $a_{m}=1$ ).

Let $B$ be a p-base for $K$ over $k$ such that $\theta$ is contained in $B$ ([3]). Then there exists one and only one derivation $\varphi$ of $K$ over $k$ such that, for $\beta \in B$,

$$
\varphi(\beta)= \begin{cases}1 & (\beta=\theta) \\ 0 & (\beta \neq 0) .\end{cases}
$$

Then there is a one-parameter family of deformations of $K$;

$$
f_{t}(a, b)=a b+t S q_{p} \varphi(a, b)+\cdots,
$$

where $S q_{p} \varphi=-\frac{1}{p} \delta \varphi^{p}=\sum_{i=1}^{p-1} \frac{1}{p}{ }_{p} C_{i} \varphi^{p-i} \cup \varphi^{i}$. We assume that $t_{t}$ is trivial, i.e., 


$$
f_{t}(a, b)=\Phi_{t}^{-1}\left(\Phi_{t} a \cdot \Phi_{t} b\right)
$$

where

$$
\Phi_{t}(a)=a+t \varphi_{1}(a)+\cdots \cdot
$$

By this assumption we obtain $\delta \varphi_{1}=S q_{p} \varphi$.

The following lemma is trivial.

Lemma 1. Let $\theta, \varphi$ be as above. Then

$$
\begin{aligned}
& S q_{p} \varphi\left(\theta^{t}, \theta\right)=\varphi^{p-1}\left(\theta^{t}\right) \\
& = \begin{cases}-\theta^{(n-1) p} & (t=n p-1 \text { for some integer } n) \\
0 & \text { (there is no integer } n \text { such that } t=n p-1) .\end{cases}
\end{aligned}
$$

Lemma 2. Let $\theta, \varphi$ be as above and $\varphi_{1}$ be a k-linear mapping of $K$ into itself such that $\delta \varphi_{1}=S q_{p} \varphi$. Then,

$$
\varphi_{1}\left(\theta^{t}\right)=t \theta^{t-1} \varphi_{1}(\theta)+n \theta^{t-p},
$$

for $n p \leqq t<(n+1) p$. In particular,

$$
\varphi_{1}\left(\theta^{n p}\right)=n \theta^{(n-1) p} .
$$

Proof. We shall prove this by induction on $t$.

1) The case $t=0$.

$$
\delta \varphi_{1}(1,1)=S q_{p} \varphi(1,1)=0 .
$$

Therefor $\varphi_{1}(1)=0$.

2) The case $t=n p$.

$$
\begin{aligned}
\varphi_{1}\left(\theta^{t}\right)= & \theta^{t-1} \varphi_{1}(\theta)+\theta \varphi_{1}\left(\theta^{t-1}\right)-S q_{p} \varphi\left(\theta^{t-1}, \theta\right) \\
= & \theta^{t-1} \varphi_{1}(\theta)+\theta\left\{(t-1) \theta^{t-2} \varphi_{1}(\theta)+(n-1) \theta^{t-1-p}\right\} \\
& -\varphi^{p-1}\left(\theta^{t-1}\right) \\
= & t \theta^{t-1} \varphi_{1}(\theta)+n \theta^{t-p}=n \theta^{(n-1) p} .
\end{aligned}
$$

3) The case $n p<t<(n+1) p$.

$$
\begin{aligned}
\varphi_{1}\left(\theta^{t}\right)= & \theta^{t-1} \varphi_{1}(\theta)+\theta\left\{(t-1) \theta^{t-2} \varphi_{1}(\theta)+n \theta^{t-1-p}\right\} \\
& -\varphi^{p-1}\left(\theta^{t-1}\right) \\
= & t \theta^{t-1} \varphi_{1}(\theta)+n \theta^{t-p} .
\end{aligned}
$$

This ends the proof. 
By Lamma 2, we have

$$
\varphi_{1}\left(\theta^{m p}\right)=m \theta^{(m-1) p} .
$$

On the other hand,

$$
\varphi_{1}\left(\theta^{m p}\right)=\varphi_{1}\left(\sum_{i=0}^{m-1} a_{i} \theta^{i p}\right)=\sum_{i=1}^{m-1} i a_{i} \theta^{(i-1) p} .
$$

Therefore $m \equiv 0(\bmod p)$ and if $a_{i} \neq 0$, then $i \equiv 0(\bmod p)$. Hence $\theta$ is an inseparable element of exponent $\geq 2$ over $k$. This is a contradiction, and we have obtained the following

Theorem. Let $K$ be an extension field of a field $k$ of characteristic $p \neq 0$. If there exists an inseparable algebraic element of exponent one over $k$ which is not contained in $k\left(K^{p}\right)$, then $K$ is not rigid, and more precisely a non-trivial integrable element of $H_{c}^{2}(K, K)$ is found in the image of $S q_{p}$.

\section{REFERENCES}

[1] M. Gerstenhaber, On the deformation of rings and algebras, Ann. of Math. 79 (1964), $59-103$.

[2] D.K. Harrison, Commutative algebras and cohomology, Trans. Amer. Math. Soc., 104 (1962), 191-204.

[3] N. Jacobson, Lecture in abstract algebra III, Van Nastrand, 1964.

\section{Nagoya University}

